\title{
Estudo de Eventos - Análise das decisões do Conselho Administrativo de Defesa Econômica em fusões e aquisições nos setores de energia elétrica e telecomunicações
}

\author{
Event Study - Analysis of the decisions of the Administrative Council of Economic Defense in \\ mergers and acquisitions in the electricity and telecommunications sectors \\ Estudio de Eventos - Análisis de las decisiones del Consejo Administrativo de Defensa Económica \\ en fusiones y adquisiciones en los sectores de electricidad y telecomunicaciones
}

Recebido: 08/01/2021 | Revisado: 10/01/2021 | Aceito: 12/01/2021 | Publicado: 13/01/2021

\author{
Julie Lima Ferreira \\ ORCID: https://orcid.org/0000-0003-1461-2078 \\ Universidade Federal do Ceará, Brasil \\ E-mail: julie.academico@gmail.com \\ Jonathans Oliveira Campos de Araújo \\ ORCID: https://orcid.org/0000-0001-9264-3860 \\ Universidade Federal do Ceará, Brasil \\ E-mail: jnathas41@hotmail.com \\ Jade Vitor Viana \\ ORCID: https://orcid.org/0000-0002-1039-0853 \\ Universidade Federal do Ceará, Brasil \\ E-mail: jadeinformatica0@gmail.com \\ Francisco Gildemir Ferreira da Silva \\ ORCID: https://orcid.org/0000-0002-5890-3769 \\ Universidade Federal do Ceará, Brasil \\ E-mail: gildemir@gmail.com
}

\begin{abstract}
Resumo
Diante das situações que o mercado financeiro pode passar, fusões e aquisições têm grandes impactos na eficiência de mercado, como abordado na teoria clássica das finanças. Diante disso, o seguinte trabalho se baseia na Hipótese de Mercados Eficientes para a aplicação do método de Estudo de Eventos para a análise de Fusões e Aquisições (F\&A) dos setores regulados de Energia Elétrica e Telecomunicações no Brasil, a fim de verificar se as decisões de permissão das fusões por parte do Conselho Administrativo de Defesa Econômica (CADE) foram assertivas e se não tiveram impacto anticompetitivo no mercado. Para cada setor foi utilizado uma variação do método do Estudo de Eventos quanto as janelas de tempo analisadas, mas sua metodologia foi mantida. Para o setor de Energia elétrica foi contemplado as fusões de Enel x CELG, de 2017, e Enel x Eletropaulo, de 2018, e para o setor de Telecomunicações foi analisado a aquisição Telefônica x GVT, de 2018. Com a aplicação do método não há evidência de interferência no mercado competitivo, na ótica do mercado de capitais, para todos os eventos contemplados, mostrando assim que as decisões do CADE se mostraram corretas e o mercado se comportou de maneira eficiente.
\end{abstract}

Palavras-chave: Fusões e aquisições; Impacto financeiro; CADE; Setores regulados; Estudo de eventos.

\begin{abstract}
Given the situations that the financial market can go through, mergers and acquisitions have major impacts on market efficiency, as discussed in classical finance theory. Therefore, the following work is based on the Efficient Markets Hypothesis for the application of the Event Study method for the analysis of Mergers and Acquisitions (M\&A) of the regulated sectors of Electric Energy and Telecommunications in Brazil, to verify whether the decisions to allow mergers by the Administrative Council for Economic Defense (CADE) were assertive and if they had no anticompetitive impact on the market. For each sector, a variation of the Event Study method was used for the time windows analyzed, but its methodology was maintained. For the electricity sector, the mergers of Enel x CELG, 2017, and Enel x Eletropaulo, 2018, were contemplated, and for the Telecommunications sector the Telefónica x GVT acquisition of 2018 was analyzed. With the application of the method there is no evidence of interference in the competitive market, from the point of view of the capital market, for all the events contemplated, thus showing that the decisions of CADE proved correct and the market behaved efficiently.
\end{abstract}

Keywords: Mergers and acquisitions; Financial impact; CADE; Regulated sectors; Event study.

\section{Resumen}

Dadas las situaciones que el mercado financiero puede pasar, las fusiones y adquisiciones tienen un impacto importante en la eficiencia del mercado, como se discute en la teoría de las finanzas clásicas. Por lo tanto, el siguiente trabajo se basa en la Hipótesis de Mercados Eficientes para la aplicación del método de Estudio de Eventos para el 
análisis de Fusiones y Adquisiciones (F\&A) de los sectores regulados de Energía Eléctrica y Telecomunicaciones en Brasil, con el fin de verificar si las decisiones de permitir fusiones por el Consejo Administrativo de Defensa Económica (CADE) eran asertivas y si no tenían un impacto contrario a la competencia en el mercado. Para cada sector, se utilizó una variación del método de estudio de eventos para las ventanas de tiempo analizadas, pero se mantuvo su metodología. Para el sector eléctrico, se contemplaron las fusiones de Enel x CELG, 2017, y Enel x Eletropaulo, 2018, y para el sector de las telecomunicaciones se analizó la adquisición de Telefónica x GVT de 2018. Con la aplicación del método no hay indicios de interferencia en el mercado competitivo, desde el punto de vista del mercado de capitales, para todos los acontecimientos contemplados, lo que demuestra que las decisiones del CADE resultaron correctas y que el mercado se comportó de manera eficiente.

Palabras clave: Fusiones y adquisiciones; Impacto financiero; CADE; Sectores regulados; Estudio de eventos.

\section{Introdução}

Os setores de energia elétrica e telecomunicações são importantes para os países como grandes demandas para a sociedade moderna, mas grandes concentrações no mercado desses setores podem causar danos a concorrência, ao consumidor, ao desenvolvimento e ao comportamento dos mercados financeiros. De acordo com a hipótese de eficiência de mercado, Eckbo (1983) afirma que o mercado absorve as informações e as precifica, ou seja, em um mercado oligopolizado, a decisão da fusão pode impactar o valor das ações das outras empresas do mercado negativamente ou positivamente. Ademais, a quantidade de trabalhos e estudos no Brasil quanto aos estudos sobre fusões e aquisições (F\&A) no mercado ainda não tem literatura tão ampla quanto de outros países considerados desenvolvidos como Estados Unidos ou Reino Unido.

Devido a isso, o presente trabalho foi motivado a estudar três fusões, duas do setor de energia elétrica no mercado de distribuição de energia e uma do setor telecomunicações no cenário brasileiro: ENEL com CELG; ENEL com ELETROPAULO e a aquisição da GVT pela Telefônica Brasil. O estudo pretende avaliar se as fusões autorizadas pelo Conselho Administrativo de Defesa Econômica - CADE foram anticompetitivas ou não no mercado financeiro. Vale ressaltar que a escolha dos dois setores se deu por se restringir a setores regulados mais consolidados na Bolsa.

Foi utilizado o método de estudo de eventos, proposto por Eckbo (1983) e reproduzido por Fernandes (2014) para as 3 análises. Para as aquisições da ENEL foram utilizados dados de: 14 de fevereiro de 2017, a qual se iniciou o processo de fusão entre ENEL e CELG; e 04 de julho de 2018, data a qual se iniciou o processo de fusão entre ENEL e ELETROPAULO, com janelas de estudo do evento $(-15,15)$ e $(-10,5)$ para ambas as aquisições. Foi utilizado para essa analise a média aritmética e ponderada dos retornos diários das rivais para utilizar os dados como amostra para regressão aplicada.

Para a aquisição da GVT pela Telefônica Brasil foi usado dados dos dias: 28 de agosto de 2014, data de anúncio da aquisição, 19 de setembro de 2014, data de confirmação de aquisição, 22 de dezembro de 2014, data de aprovação pela Anatel e 25 de março de 2015, aprovação pelo CADE, testando janelas de 5 e 20 dias, analisando o efeito, isoladamente, de cada uma dessas informações. Foi utilizado para essa analise a média aritmética dos retornos diários para a regressão aplicada. Ademais, foi analisado os retornos anormais e retornos anormais acumulados das empresas analisadas.

Diante do exposto, todos os presentes testes foram feitos para identificar se as fusões foram pró ou anticompetitivas, as janelas do teste foram definidas com foco nos efeitos de anúncio de fusão em os preços de ativos negociados na Bm\&fBovespa.

\section{Fundamentação Teórica}

\subsection{Teoria dos mercados eficientes e Hipótese de Ondas de Fusões e Aquisições}

$\mathrm{O}$ argumento principal para o uso metodológico do trabalho se baseia na teoria dos mercados eficientes (EMH) e as expectativas racionais, onde os preços das ações refletem a informação pública disponível sobre os futuros lucros das empresas. Se caso os retornos observados no mercado apresentem ausência de padrões comportamentais no mercado é observado uma anomalia, que pode ser analisada e extrair informações de prejuízos ao mercado e consequentemente a competição de mercado, 
no caso analisado de F\&A.

Como apresentado por Camargos e Barbosa (2007), há três formas de eficiência do mercado de capitais propostos por Roberts (1967) e Fama (1970), sendo elas:

- fraca, com preços que refletem completamente as informações sobre o histórico do comportamento dos preços dos ativos;

- semiforte, com preços que refletem o histórico do comportamento dos preços e todas as informações que são publicamente disponíveis (como notícias da empresa, balanços de desempenho etc.);

- forte, com preços que refletem todas as informações disponíveis (históricas, públicas e privadas).

Baseado nessa hipótese, utilizando o cenário de eficiência semiforte, é possível fazer a análise pelo Estudo de Eventos que tal evento se mostrou prejudicial para aquela firma, acionistas ou setor baseando-se no nível do impacto da anomalia detectada, no caso, sobre os retornos da empresa.

A Hipótese de Ondas (Souza \& Gartner, 2019; Pessanha, 2010) é baseada que as decisões para as empresas se fundirem não nascem dos ganhos econômicos com a fusão ou aquisição, mas sim na tendência do mercado em se torna mais concentrado. Isso ocorre, de acordo com o trabalho de Song e Walkling (2000), pois os acionistas antecipam os eventos de F\&A e aquecem o mercado acionário das empresas que possivelmente passaram por esses eventos. Esse aquecimento especulativo pode ter efeitos positivos ou negativos ao mercado e o Estudo de Eventos é um modelo para medir o nível desse aquecimento para as empresas rivais do setor e para o mercado acionário.

\subsection{Estudo de Eventos, análise de Fusões e Aquisições e sua importância}

O conceito de fusão para Rossetti (2001), "União de duas ou mais companhias que formam uma única empresa, geralmente sob controle administrativo da maior ou mais próspera" e para Marvis e Marks (1998, apud Pereira, 2007), "Envolvem a criação de uma organização, a partir da combinação de duas ou mais empresas, com nova identidade. As empresas que lhe deram origem deixam de existir legalmente".

As aquisições, para a literatura, têm como definição "Compra de controle acionário de uma empresa por outra" (Rossetti, 2001) e "Uma organização adquire o controle das ações de outra organização ou diretamente de seus proprietários" (Harrison, 2004).

Esses eventos podem causar oscilações dentro do mercado financeiro, consequentemente afetando investidores e consumidores causando perda de bem estar e utilidade, por isso a importância de estudos quanto a esse assunto.

O primeiro trabalho utilizando a aplicação do Estudo de Eventos foi Eckbo (1983), onde o autor argumenta que fusões anticompetitivas são boas não apenas para as empresas dentro do evento, mas também para suas rivais de setor, pois uma elevação de preços da nova firma formada permite que suas rivais elevem também o preço de seus produtos. Uma F\&A será considerada anticompetitiva se houver elevação de preços de ações das empresas rivais, para mensurar esse efeito nos preços é utilizado a análise dos retornos anormais.

$$
A R_{i t}=R_{i t}-\widehat{R}_{i t}=R_{i t}-\left(\hat{\alpha}_{i}+\hat{\beta}_{i} R_{m t}\right)
$$

Onde o cálculo dos retornos anormais é criado após a aplicação de uma estimação de Mínimos Quadrados Ordinários com o CAPM ou um modelo APT.

$$
R_{i t}=\alpha_{i}+\beta_{i} \cdot R_{m t}+\epsilon_{i t}
$$

Assim, sendo possível estimar os retornos anormais por meio da equação: 


$$
A R_{i t}=R_{i t}-\left(\alpha_{i}+\beta_{i} \cdot R_{m t}\right)
$$

Desta forma é possível mensurar e interpretar se há existência de retorno anormal ou não, e saber qual o impacto do evento no mercado, sobre o desempenho das ações da empresa em processo de F\&A.

Para averiguação de dados e sua confiança, é necessário também a realização de um teste de hipótese para que os dados encontrados sejam estatisticamente relevantes.

$$
\begin{aligned}
& H_{0}: A R_{i t}=0-\text { Não há retorno anormal } \\
& H_{1}: A R_{i t} \neq 0-\text { Há retorno anormal }
\end{aligned}
$$

\subsection{CADE - Agência Reguladora}

No Brasil, a autarquia federal responsável pelo monitoramento e aprovação ou, se necessário, barramento de fusões, aquisições e outras medidas administrativas da área, é o CADE, Conselho Administrativo de Defesa Econômica. Tendo o dever de zelar pela livre concorrência no mercado, executando investigações e decisões sobre a matéria de concorrência em todo o território nacional, vinculado ao Ministério da Justiça.

De acordo com a Lei $\mathrm{n}^{\circ}$ 12.529, de 30 de novembro de 2011, e complementadas pelo Regimento Interno do CADE (RiCade), o CADE exerce as seguintes funções listadas em seu site oficial sobre suas competências:

\footnotetext{
"Preventiva: analisar e posteriormente decidir sobre as fusões, aquisições de controle, incorporações e outros atos de concentração econômica entre grandes empresas que possam colocar em risco a livre concorrência

Repressiva: investigar, em todo o território nacional, e posteriormente julgar cartéis e outras condutas nocivas à livre concorrência.

Educativa: instruir o público em geral sobre as diversas condutas que possam prejudicar a livre concorrência; incentivar e estimular estudos e pesquisas acadêmicas sobre o tema, firmando parcerias com universidades, institutos de pesquisa, associações e órgãos do governo; realizar ou apoiar cursos, palestras, seminários e eventos relacionados ao assunto; editar publicações, como a Revista de Direito da Concorrência e cartilhas." (COMPETÊNCIAS..., 2011)
}

Diante do exposto, mostra-se a importante deste artigo diante da avaliação da decisão do CADE pela importância das 3 fusões analisadas para o mercado. Contudo, é importante ressaltar que no Brasil, não há apenas o monitoramento desse agente regulador para F\&A dependendo do setor, como para o setor de Energia Elétrica onde a Agência Nacional de Energia Elétrica - ANEEL também faz avaliações sobre possíveis F\&A para garantia da boa prestação de serviços a população, ressaltado por Manoel et al. (2018). Porém, no seguinte trabalho foi levado em consideração apenas os eventos de avaliação do CADE.

\subsection{Trabalhos anteriores e resultados encontrados}

O uso de Estudos de Eventos para análise de fusões e impactos no mercado é bem vasto na literatura acadêmica, e o método, inicialmente proposto por Eckbo (1983) foi várias vezes replicado ou adaptado dependendo do setor ou empresas analisadas.

No trabalho de Fernandes (2014), foram analisadas 4 fusões, Santander x Real (2007), Itaú × Unibanco (2008), Gol × Webjet (2011) e Trip $\times$ Azul (2012), onde foi observado os retornos de uma carteira composta por ações das empresas rivais de cada caso analisado com uma distância de ao menos 30 dias de negociação em bolsa para cada evento analisado. Nele foi encontrado que a decisão do CADE ao permitir a fusão foi assertiva, sendo o efeito líquido das fusões foi identificado como 
nulo.

No artigo de Camargos e Barbosa (2007) foram analisados 32 processos de fusões e aquisições ocorridos entre 1994 e 2001 no mercado de capitais brasileiro, utilizando a técnica do estudo de eventos na forma logarítmica. Foi feito um teste de hipóteses tal qual proposta por Eckbo e um teste de hipóteses de verificação da diferença de medias dos retornos anormais do período anterior e posterior a F\&A, sendo estatisticamente diferente e maior no período a média do retorno anormal, seria encontrado a criação de valor da empresa para os acionistas. Concluiu-se que o mercado se comportou de maneira eficiente durante das F\&A analisadas para análise de HME e para a análise de criação de valor para os acionistas foi sinalizado que não correu, principalmente para as empresas adquiridas.

No trabalho de Li e Lucinda (2009), foi analisada a fusão da Oi com a Brasil Telecom (BrT) com base em uma seleção e formação de uma carteira com todas suas rivais de telefonia fixa e telefonia móvel e foi utilizado os índices ITEL, índice calculado pela Bovespa do setor de telefonia e o índice Dow Jones Telecommunication Titans Index, das 30 maiores empresas de telefonia para a regressão de análise. Foram analisados 3 eventos, 3 dias representativos das etapas da fusão, sendo eles: 10/04/2007, 09/01/2008 e 25/04/2008. O método utilizado foi muito semelhante ao de Eckbo (1983), se diferenciado no procedimento de teste de retornos anormais acumulados (AAR). Seus resultados indicaram que os efeitos da fusão causaram maior competição sobre as rivais de menor participação no mercado de telefonia fixa e de menor competição para as rivais já consolidadas na telefonia móvel.

Ademais, no trabalho de Muro (2014), usando a metodologia de Mackinlay (1997) de estudo de eventos, analisou 11 fusões e aquisições dentro do setor de telecomunicações brasileiro. Onde seus resultados mostraram que as transações não foram precificadas no mercado na dada do anúncio, ou seja, o peso da divulgação das fusões não foi repassado para os preços das ações analisadas. Sendo assim, não foi comprovado uma ação anticompetitiva no objeto de estudo, pois não foi possível concluir que houve existência de retornos anormais nas fusões e aquisições analisadas do setor de telecomunicação brasileiro.

\section{Metodologia}

O método de Estudo de Eventos, proposto inicialmente por Eckbo (1983) e reproduzido no trabalho com base em Fernandes (2014), consiste em analisar o impacto do evento estudado para o mercado de ações, através da observação do nível de significância dos retornos anormais das empresas rivais, já que pela hipótese de eficiência de mercado, os agentes econômicos com base em suas expectativas, precificam a nova informação que pode impactar positivamente ou negativamente os preços das ações das outras empresas do setor.

Sendo considerado um estudo quantitativo, onde Pereira et al. (2018, p. 69) explica que esse tipo de estudo trata com coleta de dados numéricos que geral conjuntos ou massas de dados que podem ser analisados por meio de técnicas matemáticas, no presente artigo o Estudo de Eventos, na qual podem trazer a possibilidade de previsão dos acontecimentos, além de trazer embasamento firmes a estudos de caso.

No presente trabalho o evento analisado trata-se do anúncio de uma fusão para o mercado e busca-se analisar os impactos dos efeitos unilaterais das fusões nos preços das ações de suas concorrentes no mercado. Através da regressão que terá a seguinte estrutura:

$$
\mathrm{Rc}=\alpha+\beta \mathrm{Rm}
$$

Rc: retorno da carteira das rivais

Rm: Retorno do Ibovespa

Calcula-se os retornos anormais (RA) através da diferença entre o retorno estimado e o retorno efetivo através da fórmula:

$$
\mathrm{RA}=\mathrm{Yi}-(\alpha+\beta \mathrm{Rm})
$$

Os retornos anormais acumulados (RAAC) são calculados através do somatório de todos os retornos anormais como 
mostrado a seguir:

$$
\mathrm{RAAC}=\sum_{t=1}^{T} R A
$$

A variância dos retornos anormais é próxima à variância da regressão estimada, pois há um número muito grande de dados, então o segundo termo tende a zero. Nesse trabalho será usada a aproximação.

$$
\sigma^{2} R A=\sigma^{2}+\frac{1}{L 1}\left[1+\frac{R m-\mu m}{\sigma^{2} m}\right]
$$

$$
\begin{array}{cc}
\text { L1: tamanho da janela de estimação } & \sigma^{2} \text { m: variância de } \mathrm{Rm} \\
\mu \mathrm{m} \text { : média de } \mathrm{Rm} & \sigma^{2} \text { : variância da regressão }
\end{array}
$$

Já a variância dos retornos anormais acumulados é estimada da seguinte maneira:

$$
\sigma^{2} \mathrm{RAAC}=\mathrm{L} 2 \sigma^{2}
$$

L2: número de dias da janela do evento

Para os testes de significância são usados testes t's, com 5\% de nível de significância e com as variâncias já estimadas fazemos o teste da seguinte forma:

$$
\begin{aligned}
t R A & =\frac{R A}{\sigma} \\
t R A A C & =\frac{R A A C}{\sigma R A A C}
\end{aligned}
$$

A partir do teste estatístico, é verificado se os retornos anormais das rivais foram significantes, ou seja, se o evento em questão teve efeito sobre o mercado, para verificar a Hipótese de Mercados Eficientes. Além disso, através da teoria das fusões, pode-se definir se a fusão foi anticompetitiva ou pró competitiva, tomando como base o sinal do teste t. A seguir temos o processo metodológico utilizado no trabalho de forma prática:

Tabela 1. Processo metodológico utilizado no trabalho.

\section{Processo metodológico do trabalho resumido}

1. Identificação das datas dos eventos;

2. Identificação das empresas rivais, para os dois setores;

3. Definição das datas e a janela de análise para cada setor;

4. Coleta de dados e cálculo dos retornos diários que serão analisados;

5. Realizar a regressão simples por MQO dos retornos diários em relação ao índice de mercado, considerando as janelas estabelecidas para cada setor;

6. Cálculo dos Retornos Anormais e Retornos Anormais Acumulados;

7. Teste de significância $t$ com hipótese nula de que os retornos anormais são iguais a zero, ou seja, que o evento em estudo não tem impacto sobre as firmas analisadas.

Fonte: Autores.

\subsection{Setor de Energia Elétrica}

As fusões contempladas pela análise foram: ENEL e CELG; ENEL e ELETROPAULO. Foram feitas carteiras com as principais rivais das empresas, que foram selecionadas com base no critério de receita do ano anterior à data da operação de fusão. A regressão do estudo de eventos foi feita com base nas medias aritméticas e ponderadas da carteira criada para fins comparativos.

O evento analisado foi a data em que se deu início à operação de fusão das merging firms. E para os dois casos foram 
utilizadas as janelas de $(-15,15)$ e $(-10,5)$, ou seja, 15 dias antes do evento e 15 dias após o evento e outra para 10 dias antes e 5 dias depois do evento.

Para os dois casos, os dados utilizados para regressão foram uma média aritmética dos retornos das rivais e uma média ponderada pelo percentual da receita de cada rival em relação ao somatório da receita das rivais escolhidas.

\subsubsection{ENEL x CELG}

A seleção das principais rivais das merging firms tomou como base a receita das distribuidoras de energia no ano de 2016. As rivais selecionadas podem ser vistas na Tabela 2.

Tabela 2. Principais rivais das merging firms.

EMPRESA

RECEITA

PESO RELATIVO

\begin{tabular}{ccc}
\hline CEMIG-D - CEMIG DISTRIBUIÇÃO S. A & $\mathrm{R} \$ 11.836 .671 .546,93$ & $30 \%$ \\
\hline LIGHT - LIGHT SERVIÇOS DE ELETRICIDADE S. A & $\mathrm{R} \$ 10.149 .643 .292,71$ & $25 \%$ \\
\hline COPEL-DIS - COPEL DISTRIBUIÇÃO S. A & $\mathrm{R} \$ 9.303 .838 .005,33$ & $23 \%$ \\
\hline CPFL-PAULISTA - COMPANHIA PAULISTA DE FORÇA E LUZ & $\mathrm{R} \$ 8.786 .866 .126,38$ & $22 \%$ \\
\hline TOTAL & $\mathbf{R} \$ \mathbf{4 0 . 0 7 7 . 0 1 8 . 9 7 1 , 3 5}$ & $\mathbf{1 0 0 \%}$ \\
\hline
\end{tabular}

Fonte: Autores.

Observa-se que a empresa CEMIG é a maior rival do mercado, contudo as participações das empresas na carteira de análise foram parecidas no mercado.

A data utilizada para análise foi o dia 14/02/2017, a qual se iniciou o processo de fusão entre as duas empresas. Após a seleção das principais rivais foi feita a média aritmética e ponderada dos retornos diários das rivais para utilizar os dados como amostra para regressão. Foi utilizada uma amostra de 200 observações com uma distância de 50 dias da data do evento estudado para realizar a regressão dos retornos médios das rivais em relação ao índice Ibovespa.

Após isso, foram definidas as janelas de estudo do evento (15:15) e (10:5) e para cada dia da janela do evento foram calculados os retornos estimados através do valor dos coeficientes encontrados, conforme modelo abaixo.

$$
R_{M}=\alpha+\beta X+\epsilon
$$

Onde o retorno médio das rivais é explicado pelo índice de mercado Ibovespa e um distúrbio aleatório.

No método do estudo de eventos, busca-se analisar a anormalidade do distúrbio aleatório que é a parcela não explicada pelo índice Ibovespa. Dessa forma, assume-se que o erro na equação captura a informação do evento estudado.

Para encontrar os retornos anormais, portanto, foi calculada a diferença entre os retornos efetivos e estimados. Por fim, a fim de determinar a significância dos retornos anormais, foi feito um teste $t$.

\subsubsection{ENEL $x$ ELETROPAULO}

A seleção das principais rivais das merging firms tomou como base a receita das distribuidoras de energia no ano de 2017. As rivais selecionadas podem ser vistas na Tabela 3. 
Tabela 3. Principais rivais das merging firms.

EMPRESA

RECEITA

PESO RELATIVO

\begin{tabular}{ccc}
\hline CEMIG-D - CEMIG DISTRIBUIÇÃO S. A & $\mathrm{R} \$ 11.552 .549 .202,95$ & $31 \%$ \\
\hline LIGHT - LIGHT SERVIÇOS DE ELETRICIDADE S. A & $\mathrm{R} \$ 9.396 .949 .186,47$ & $25 \%$ \\
\hline CPFL-PAULISTA - COMPANHIA PAULISTA DE FORÇA E LUZ & $\mathrm{R} \$ 8.261 .157 .299,11$ & $22 \%$ \\
\hline COPEL-DIS - COPEL DISTRIBUIÇÃO S. A & $\mathrm{R} \$ 7.952 .611 .580,06$ & $21 \%$ \\
\hline TOTAL & $\mathrm{R} \$ 37.163 .267 .268,59$ & $100 \%$ \\
\hline
\end{tabular}

Fonte: Autores.

Observa-se que a composição da carteira das empresas rivais tem composição diferente da carteira do evento estudado anterior, porém as participações, embora diferentes, têm peso parecido no mercado.

A data utilizada para análise foi o dia 04/06/2018, a qual se iniciou o processo de fusão entre as duas empresas.

Após a seleção das principais rivais foi feita a média aritmética e ponderada dos retornos diários das rivais para utilizar os dados como amostra para regressão.

Foi utilizada uma amostra de 200 observações com uma distância de 50 dias da data do evento estudado para realizar a regressão dos retornos médios das rivais em relação ao índice Ibovespa.

Após a coleta dos dados foi feita o mesmo procedimento descrito na fusão ENEL x CELG.

\subsection{Setor de Telecomunicações}

O evento a ser analisado será a aquisição da GVT pela Telefônica Brasil (VIVT3), porém será usado quatro datas diferentes: o anúncio da oferta pela aquisição em 28/08/2014, o anúncio da aquisição em 19/09/2014, a permissão da aquisição pela Anatel em 22/12/2014 e a permissão do CADE em 25/05/2015. Em relação às empresas rivais, serão analisados os efeitos decorrentes desse evento na Oi S.A. (OIBR3) e na Algar Telecom S.A. (ALGT), pois elas seriam as mais afetadas devido à semelhança entre seus serviços prestados aos da GVT.

O dia do evento será mencionado como dia 0 e a janela do evento será $(-5,5)$, isso é, 5 dias antes a 5 dias depois do evento e a janela $(-20,20), 20$ dias antes e 20 dias depois. Será estimada uma regressão diferente para cada evento observado, além dos respectivos retornos anormais e retornos anormais acumulados, e será analisado se houve alguma variação fora do padrão nos dias que rondaram o evento. No total serão estimadas oito regressões diferentes.

Para estimar os parâmetros, será usado o modelo de Mínimos Quadrados Ordinários (MQO), sendo o retorno da carteira de empresas rivais, calculado através da média aritmética dos retornos das duas ações, a variável dependente e o retorno do Ibovespa como variável independente. Diferentemente da análise para setor de Energia Elétrica, não feito uma análise com cálculo através de média ponderada por serem apenas duas rivais contempladas. A regressão usará os dados de até 5 e 20 dias antes data do evento, esse método é usado para que o período ao redor da aquisição não afete a regressão do período normal. Serão usadas 184 observações em cada regressão estimada.

Os períodos serão chamados de: "Evento 1" para marcar o dia do anúncio da oferta pela GVT, "Evento 2" que marcou o dia da confirmação da aquisição, "Evento 3" marcará a permissão da aquisição pela Anatel e "Evento 4" como o dia da permissão concedida pelo CADE. 


\section{Resultados e Discussão}

Para a realização das regressões foi utilizado dois métodos para a elaboração das carteiras das rivais analisadas, com média aritmética e média ponderada, assim obteve-se os seguintes resultados apresentados na Tabelas 4 e 5:

Tabela 4. Resultados da regressão dos retornos médios das rivais em relação ao Ibov - Média Aritmética.

\section{Resultados da regressão utilizando média aritmética}

\begin{tabular}{ccccc}
\hline- & Coeficientes & Erro padrão & valor-P & Teste t \\
\hline Intercepto & $-0,00004088$ & 0,00114308 & 0,97150650 & $-0,035764086$ \\
& & & & \\
\hline Variável IBOV & 1,03914361 & 0,06828884 & 0,00000000 & 15,2168876 \\
& & & & \\
\hline
\end{tabular}

Fonte: Autores.

Tabela 5. Resultados da regressão dos retornos médios das rivais em relação ao Ibov - Média Ponderada.

\section{Resultados da regressão utilizando média ponderada}

\begin{tabular}{ccccc}
\hline- & Coeficientes & Erro padrão & valor-P & Teste t \\
\hline Intercepto & $-0,000116385$ & 0,001174806 & 0,921184884 & $-0,099067522$ \\
& & & 0,000 & 15,198818716 \\
\hline Variável IBOV & 1,066713406 & 0,070183968 & & \\
\hline
\end{tabular}

Fonte: Autores.

A partir da regressão feita e seu teste $t$, teve-se resultados que mostraram que para as duas janelas do evento, tanto utilizando média ponderada, como média aritmética, a fusão da ENEL e CELG não foi significante estatisticamente e, portanto, não teve influência sobre o preço das ações das outras empresas do setor. As duas regressões foram feitas para fins comparativos e seus resultados foram parecidos.

Com os dados da regressão foi feito, portanto, os retornos anormais os eventos para o dia 14/02/2017 são apresentados na Tabela 6:

Tabela 6. Resultado dos retornos anormais para análise ENEL x CELG.
Técnica Utilizada
Janelas dos eventos
RAAC
RAAC test t

$\begin{array}{lll}\mathbf{( - 1 5 , 1 5 )} & 0,1610 & 1,7563\end{array}$

Média Aritmética

$\mathbf{( - 1 0 , 5 )} \quad 0,1036 \quad 1,6172$

Média Ponderada

$\mathbf{( - 1 5 , 1 5 )} \quad 0,1610 \quad 1,7563$

$\begin{array}{lll}\mathbf{( - 1 0 , 5 )} & 0,1029 & 1,5617\end{array}$


Os resultados encontrados nos testes $\mathrm{t}$ da criação dos retornos anormais para fusão ENEL $\mathrm{x}$ CELG não foram significantes, logo, essa fusão não se mostrou influente no mercado de Energia Elétrica na bolsa de valores.

\subsection{ENEL x ELETROPAULO}

Os resultados da regressão podem ser vistos a seguir na Tabelas 7 e 8.

Tabela 7. Resultado da regressão dos retornos médios das rivais em relação ao IBOV - Média Aritmética.

\section{Resultados da regressão utilizando média aritmética}

\begin{tabular}{ccccc}
\hline- & Coeficientes & Erro padrão & p-valor & Teste t \\
\hline Intercepto & $-0,0013$ & 0,0008 & 0,1114 & $-1,5990$ \\
\hline Variável IBOV & 0,7020 & 0,0780 & 0,0000 & 8,9996 \\
\hline
\end{tabular}

Fonte: Autores.

Tabela 8. Resultados da regressão dos retornos médios das rivais em relação ao Ibov - Média Ponderada.

\section{Resultados da regressão utilizando média ponderada}

\begin{tabular}{ccccc}
\hline- & Coeficientes & Erro padrão & p-valor & Teste t \\
\hline Intercepto & $-0,0014$ & 0,0008 & 0,1102 & $-1,6047$ \\
\hline Variável IBOV & 0,7259 & 0,0806 & 0,0000 & 9,0115 \\
\hline
\end{tabular}

Fonte: Autores.

Observa-se que os resultados entre as regressões foram parecidos com média aritmética e média ponderada. E o teste $\mathrm{t}$ dos interceptos apresentou valores significantes estatisticamente.

Os retornos anormais foram calculados dentro das janelas $(-15,15)$ e $(-10,5)$. Sendo os resultados para o dia do evento, 04/06/2018, apresentados pela Tabela 9:

Tabela 9. Resultado dos retornos anormais para análise ENEL x ELETROPAULO.

Técnica Utilizada

Média Aritmética

Média Aritmética
RAAC

$\mathbf{( - 1 5 , 1 5 )}-0,0142 \quad-6,8960$

$\begin{array}{lll}\mathbf{( - 1 0 , 5 )} & 0,0353 & 0,7720\end{array}$

$\begin{array}{lll}\mathbf{( - 1 5 , 1 5 )}-0,0129 & -6,0706\end{array}$

Média Ponderada

$\begin{array}{lll}\mathbf{( - 1 0 , 5 )} & 0,0350 & 0,7414\end{array}$

Fonte: Autores.

$\mathrm{O}$ resultado do teste $\mathrm{t}$ dos retornos anormais acumulados considerando a janela (10:5) foi insignificante 
estatisticamente. Já no teste t para a janela (15:15) foi possível identificar que a informação sobre o processo de fusão impactou negativamente os retornos das rivais, e, portanto, a princípio, considerando que o mercado é eficiente, pode-se concluir que a fusão da ENEL X ELETROPAULO foi pró competitiva, ou seja, houve ganhos de eficiência nos custos marginais.

\subsection{Setor de Telecomunicações}

Utilizando a metodologia anteriormente explicada, estima-se os seguintes resultados para os 4 eventos analisados em 8 regressões apresentados a seguir na Tabela 10:

Tabela 10. Resultados das regressões para os eventos da fusão GVT x Telefônica.

\section{Resultados das Regressões para Setor de Telecomunicações}

\begin{tabular}{|c|c|c|c|c|c|}
\hline- & Coeficientes & Valor Estimado & Desvio Padrão & Teste $\mathbf{t}$ & P-Valor \\
\hline & Alfa & $-0,0015$ & 0,0024 & $-0,6481$ & 0,5187 \\
\hline & Beta & 0,8375 & 0,1955 & 4,2840 & $0,0001 * * *$ \\
\hline & Alfa & $-0,0020$ & 0,0017 & $-1,2090$ & 0,2283 \\
\hline & Beta & 0,5246 & 0,1409 & 3,7240 & $0,0003 * * *$ \\
\hline & Alfa & $-0,0018$ & 0,0017 & $-1,0740$ & 0,2844 \\
\hline & Beta & 0,4485 & 0,1412 & 3,1760 & $0,0018 * * *$ \\
\hline & Alfa & $-0,0022$ & 0,0017 & $-1,2910$ & 0,1984 \\
\hline & Beta & 0,4654 & 0,1431 & 3,2530 & $0,0014 * * *$ \\
\hline \multirow{2}{*}{ Evento $3(-5,5)$} & Alfa & $-0,0021$ & 0,0017 & $-1,2240$ & 0,2226 \\
\hline & Beta & 0,4274 & 0,1025 & 4,1710 & $0,0001 * * *$ \\
\hline \multirow{2}{*}{ Evento $3(-20,20)$} & Alfa & $-0,0016$ & 0,0017 & $-0,9514$ & 0,3427 \\
\hline & Beta & 0,3989 & 0,1054 & 3,7860 & $0,0002 * * *$ \\
\hline \multirow{2}{*}{ Evento $4(-5,5)$} & Alfa & $-0,0012$ & 0,0019 & $-0,6121$ & 0,5412 \\
\hline & Beta & 0,4126 & 0,1075 & 3,8380 & $0,0002 * * *$ \\
\hline \multirow{2}{*}{ Evento $4(-20,20)$} & Alfa & $-0,0021$ & 0,0019 & $-1,1160$ & 0,2660 \\
\hline & Beta & 0,3877 & 0,1087 & 3,5660 & $0,0005 * * *$ \\
\hline
\end{tabular}


Reportado na Tabela 10, em todas as regressões o beta de mercado da carteira, formada por Oi S.A. e Algar Telecom, foi significante a um nível de $1 \%$ de significância e os alfas não, indicando não haver evidências que o ativo ou carteira de ativos superou a expectativa de rendimento prevista para ele. Vale ressaltar que o Alfa do CAPM apresentado nos resultados tem a interpretação do valor esperado do ativo desconsiderando o mercado.

Depois desses resultados, pode ser feito a análise dos retornos anormais acumulados para analisar se o evento trouxe alguma alteração no comportamento das ações. Os resultados são apresentados na Tabela 11:

Tabela 11. Resultado dos retornos anormais para análise GVT x Telefônica.

\section{Eventos}

\begin{tabular}{llc}
\hline Evento $1(\mathbf{- 5 , 5})$ & $9,67 \%$ & 1,3525 \\
\hline Evento $\mathbf{1}(\mathbf{- 2 0 , 2 0})$ & $17,92 \%$ & 1,2235 \\
\hline Evento 2 (-5,5) & $7,05 \%$ & 0,9270 \\
\hline Evento 2 (-20,20) & $7,83 \%$ & 0,5345 \\
\hline Evento 3 (-5,5) & $-6,44 \%$ & $-0,8388$ \\
\hline Evento 3 (-20,20) & $-12,67 \%$ & $-0,8603$ \\
\hline Evento $4(-\mathbf{5 , 5})$ & & 0,9648 \\
\hline Evento $4(-\mathbf{2 0 , 2 0})$ & $8,18 \%$ & 0,3150 \\
\hline
\end{tabular}

Fonte: Autores.

Como observado, apenas no "Evento 3" o valor foi negativo, indicando que essa aquisição poderia se tratar de um ato anticompetitivo sobre as rivais analisadas e que os eventos 2 e 4 para janelas maiores parecem ter impacto reduzido. Porém em nenhum dos quatro eventos determinantes houve significância no valor encontrado, portanto não há indícios de distúrbio devido ao ato de concentração.

\section{Considerações Finais}

O trabalho buscou aplicar o método do estudo de eventos às fusões do setor de distribuição de energia elétrica e setor de telecomunicações para analisar se as fusões foram anticompetitivas ou pró competitivas para eventos dos setores de energia elétrica e telecomunicações e se a Anatel e o CADE acertaram na sua decisão de permitir que ela ocorresse.

Para os eventos do setor de energia elétrico, foi observado que na data da fusão da ENEL e CELG, os retornos anormais não foram significantes, ou seja, a fusão não teve grande impacto no mercado de distribuição de energia.

Já para a operação da fusão entre a ENEL e ELETROPAULO, os retornos anormais das rivais foram negativos e estatisticamente significantes, ou seja, o mercado interpretou que a fusão aumentaria a eficiência nos custos marginais.

Para o setor de telecomunicações, pode-se concluir, com base nos resultados encontrados, que esse ato de concentração de mercado não gerou grande impacto no funcionamento do setor de telecomunicações.

O resultado sugere neutralidade, podendo ser motivado pela aquisição da GVT uma vez que a Telefônica Brasil ainda 
não ofertava o serviço daquela, portanto sendo o efeito marginal na concentração insignificante na oferta dos serviços.

Diante do exposto, a Anatel e o CADE acertaram na sua decisão de permitir que essas fusões/aquisições acontecessem, baseando-se nos resultados obtidos no Estudo de Eventos realizado.

Não obstante, fica de sugestão para futuros trabalhos acadêmicos a análise de maiores janelas de observação para os eventos utilizados, até mesmo utilizando como referência do mercado o IEE (Índice de Energia Elétrica) ao invés do Índice Bovespa para as análises do setor elétrico, e para novas fusões e aquisições que poderão existir dentro desses dois grandes setores em crescimento no Brasil. Além de uma possível continuação da análise na mesma linha do trabalho de Camargos \& Barbosa (2007), para os eventos analisados neste trabalho, afim de verificar a criação de valor para os acionistas, como complemento de análise de eficiência do mercado. Ademais, é possível utilizar o trabalho de Backes e Hemkemeier (2020) como base para um estudo utilizando o método de Estudo de Eventos para o setor regulado de saneamento no Brasil com o anúncio do Novo Marco regulatório no setor.

\section{Agradecimentos}

Os autores agradecem Conselho Nacional de Desenvolvimento Científico e Tecnológico (CNPq), a Fundação de Amparo à Pesquisa do Ceará e a Universidade Federal do Ceará por financiarem o desenvolvimento deste trabalho com bolsas de PIBIC e de pesquisa.

\section{Referências}

Backes, H. S., \& Hemkemeier, M. (2020). O mercado de renda variável e as empresas do ramo do saneamento: o impacto dos investimentos associados ao Novo Marco do Saneamento. Research, Society and Development, 9(12), e38091211199. https://doi.org/10.33448/rsd-v9i12.11199

Bergmann, D. R., Savoia, J. R. F., de Melo Souza, B., \& de Mariz, F. (2015). Avaliação dos Processos de Fusões e Aquisições no setor Bancário Brasileiro por meio de Estudo de Eventos. Revista Brasileira de Gestão de Negócios-RBGN, 17(56), 1105-1115.

Camargos, M. A., \& Barbosa, F. V. (2007). Análise empírica da reação do mercado de capitais brasileiro aos anúncios de fusões e aquisições ocorridos entre 1994 e 2001. Revista de Administração, 42 (4), 468-481.

Competências - CADE. (2011). http://www.cade.gov.br/acesso-a-informacao/institucional/copy_of_competencias/capa-interna.

dos Santos, A. B., \& Perobelli, F. F. C. (2009). Reação do mercado à deliberação de planos de opção de compra de ações: um estudo de eventos para as empresas negociadas na Bovespa. Revista Brasileira de Finanças, 7(2), 163-195.

Ellwanger, K. (2014). Fusões e aquisições de empresas transnacionais: um estudo de eventos sobre o retorno ao acionista. Revista Eletrônica em Gestão, Educação e Tecnologia Ambiental, 18(1), 504-520.

Fernandes, P. R. L. (2015). Análise das decisões do CADE com uso de estudos de eventos. (Doctoral dissertation, Dissertação de Mestrado. Pós-graduação em Economia, CAEN da Universidade Federal do Ceará. Fortaleza).

Goes, J. S. (2015). Fusão Sadia/Perdigão: Análise do caso sob a metodologia de estudos de eventos.

Goldstein, C. N. (2008). Estudo de eventos: o uso de informação privilegiada em operações de fusão e aquisição de empresas brasileiras entre os anos de 1995 e 2008 .

Harrison, J. S. (2004) Administração estratégica de recursos e relacionamentos. Bookman.

Li, J. T. S., Lucinda, C. R. (2009). Potencial anticompetitivo da fusão Oi-BrT: uma análise usando retornos anormais e estudo de eventos. Revista Brasileira de Economia de Empresas, Brasília, 9 (2), 25-40.

Manoel, C. A. S., Hayashi, C., \& Silva, L. H. de A. e. (2018). A importância da ANEEL na prestação dos serviços de energia elétrica no Brasil. Research, Society and Development, 7(7), e377276. https://doi.org/10.17648/rsd-v7i7.276

Muro, P. B. (2014). Análise de fusões e aquisições no setor de telecomunicações brasileiro. (Doctoral dissertation, Monografia. Ciências econômicas, Insper Instituto de Ensino e Pesquisa. São Paulo).

Patrocínio, M. R., Kayo, E. K., \& Kimura, H. (2007). Aquisição de empresas, intangibilidade e criação de valor: um estudo de evento. Revista de Administração, 42(2), 205-215.

Pereira A. S. et al. (2018). Metodologia da pesquisa científica

Ed.

UAB/NTE/UFSM

https://repositorio.ufsm.br/bitstream/handle/1/15824/Lic_Computacao_Metodologia-Pesquisa-Cientifica.pdf?sequence=1 . 
Research, Society and Development, v. 10, n. 1, e29110111756, 2021

(CC BY 4.0) | ISSN 2525-3409 | DOI: http://dx.doi.org/10.33448/rsd-v10i1.11756

Pereira, M. A. D. S. (2007). Fusões e Aquisições. Um estudo em uma empresa nacional do Setor Elétrico (Doctoral dissertation, Dissertação de Mestrado. Administração, Faculdade Novos Horizontes. Belo Horizonte).

Pessanha, G. R. G. (2010). Os efeitos das fusões e aquisições na rentabilidade e no risco: uma análise empírica do setor bancário brasileiro no período de 1994 a 2009 (Doctoral dissertation, Dissertação de Mestrado, Universidade Federal de Lavras, Lavras, MG, Brasil).

Rossetti, J. P. (2001) Fusões e aquisições no Brasil: as raízes e os impactos. Fusões, aquisições \& parcerias. Cap. 3, 67-87.

Souza, J. G. D. M., \& Gartner, I. R. (2019). Market reaction to bank merger and acquisition events in Brazil: an analysis of the effects of market waves. Revista Contabilidade \& Finanças, (AHEAD).

Kirch, G. (2019). Estudo de Eventos, estimação do retorno esperado e as condições de mercado. ConTexto, 19(41). 